\title{
Effects of APP Software on Autonomic Physiological Responses under Different Stimulation Modes
}

\author{
Zhihai $\mathrm{Hu}^{1, \text { a }}$, Xiu $\mathrm{Wu}^{1, \mathrm{~b}}$ \\ ${ }^{1}$ School of Education Science, Huangshan University, Huangshan, China

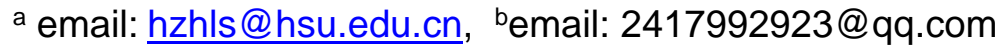

\begin{abstract}
Keywords: visual channel; audio visual pathway; smart phone; app software; autonomic physiological response.

Abstract. Objective: To explore the differences of the app software in different stimulation modes on the individual's independent physiological responses, and to analyze the differences of the responses of the subjects of different genders and grades in different stimulation modes. Methods: 51 college students were randomly selected as subjects. Each has to present subjects with six kinds of common APP software, APP software of these six kinds of images and pictures about the software and recording instrument with voice, skin temperature, blood volume pulse and skin conductance of these physiological indexes by using physiological. Results: pictures, pictures and sounds with the stimulation mode of APP software have led to the individual physiological responses, the physiological autonomic response effect of pictures with sound stimulation combined mode is better than the single channel stimulation mode picture stimulus, and these two kinds of stimuli in the skin and skin temperature significantly. Conclusion: The app software under two kinds of stimulation modes all lead to the physiological response of the individual, and the effect of the picture and sound stimulation is better than the picture stimulus.
\end{abstract}

\section{Introduction}

With the popularization and development of intelligent mobile phone mobile Internet, mobile phone and college students' life and work are inseparable, the use of APP mobile phone has an important influence on College Students' study, life, communication, entertainment, makes communication more convenient, but also to meet the information needs to obtain information, enrich students' life. The use of mobile clients has a huge impact on people, but no one has done any research in this area. Therefore, the purpose of this study is to use the physiological of physiological activity of people to use the APP software of the polygraph, then analyzes the influence of physiological activities which stimulate the mode of APP software to the maximum.

Zhao Yawei et al. (2014) study the emotional design of mobile phone APP interface, and put forward the affective factor model which affects the APP interface of mobile phone. [1] Li Ting et al. (2015) based on the user experience of smart phone APP interface design research, obtained the sensory perception experience level, emotional experience level, cognitive evaluation of the user experience structure model. [2] Wang Huan et al (2014) study the relationship between personality traits and mobile phone dependence, and conclude that social anxiety plays a partial mediating role between them. [3] Jiang Yongzhi et al. (2014) study the influence of Internet dependence on the alienation of College Students' mobile phone. [4]

In the literature, the intelligent mobile phone in recent years APP research mainly focuses on the interface design and emotional design research of APP mobile phone, the research is focused on the mobile phone dependence level, involves little physiological research on intelligent mobile phone APP, this experiment provides a new research direction.

Zheng Xifu (2003) studied the priming effect of different emotional patterns, and found that there are differences in the inhibition time between positive and negative emotions, which may be caused by the difference between the different attitudes towards the two emotions. [5] Yang Hongyu et al. (2005) the neuroendocrine changes of individual physiological indexes before and after the study of positive and negative emotional picture stimuli, the negative emotions by positive emotions, negative effects of emotion, emotional changes of two kinds of the sympathetic activity is different. [6] Ren 
Jun et al. (2012) to study the effect of meditation training on the emotional arousal of the positive and negative emotional pictures. It is concluded that meditation reduces the emotional response to the two emotional pictures, but also acts as a smoothing effect on the emotional response. [7]

Miao Yanmin et al. (2009) the difference induced by emotional patterns of different sensory channels, that visual stimulation combined with one induced by the mode is superior to visual and auditory stimuli evoked by stimulation of single channel, the different modes of emotional recovery are also different. [8]Thomas Baumgartnwe et al. (2006) with music and picture combination induction of happiness and sadness, fear of three emotions, the induced effect is the best combination in pictures and music, followed by the picture, the worst is the sound. [9] Mauri Maurizio et al. (2011) to investigate why Facebook to be so successful, and put forward the concept of core flow, namely people use Facebook in a high price and high arousal physiological and psychological state, that social networking sites such as Facebook success experience and positive emotions about this particular user. [10]

From the status of physiological research above can see the physiological aspects of research on intelligent mobile phone APP most related to the little stimulation induced materials used are related to emotion and pictures, music or video clips, but also induce the changes of mood. But most of them are in single channel stimulation mode, produce emotional reaction in life is the most with the different stimulation patterns under the condition of research in recent years has mode of binding to different stimuli induced physiological responses. However, the previous selection of emotional materials, pictures and sound of the material, although the corresponding emotions, but they are independent of each other. The experiment is selected and is closely related to the life of the APP software, pictures, pictures and sound is also related to the combination of APP software to stimulate different physiological responses under different stimulus patterns.

In addition to the experimental study on single channel picture stimulus induced by the mode of physiological reaction effect, also studied the audio-visual (picture and sound combination) stimulation effect of combination, to compare the induced physiological reaction of two kinds of different stimulation mode; the experiment through a number of physiological index continued to record standard, make people's physiological changes on their own the better understanding and further understanding of APP software in the actual use of mobile phone, what kind of stimulation mode of physiological and emotional stronger. In addition, APP software designers can learn from the results of this experiment, choose the best way of software development, not only to meet the growing consumer demand, but also get the potential commercial value from.

\section{Research Methodology}

Subjects The experiment selected a total of 51 college students, including a total of 26 male, female students, a total of 25 people, the first grade, the second grade, the second grade of the 12, the third year of the 17. Age between 18-23 years old, with an average age of 21.32. Give a certain reward after the experiment.

Materials and Instruments According to the analysis data of Harvard Business School researchers, the brains of people every day through the five senses of external information respectively: $1 \%$ taste, smell touch $1.5 \%, 3.5 \%, 11 \%$ and $83 \%$ Visual Auditory. we can see that the visual information in the acceptance of external information accounted for $81 \%$ of the proportion, the proportion of hearing accounted for $11 \%$. Therefore, select the app software images, as well as the combination of pictures and sound. Selection of APP material is 6 types higher frequency of use in the daily lives, were social class (QQ), video games (Iqiyi), (happy Diminshing Music), shopping (Taobao), payment (Alipay), music (cool dog), in addition, from the International Affective Picture System in selected neutral material pictures as picture stimuli (visual) baseline phase; neutral pictures and pictures of the stimulation, the sound selection is "quiet" this piece of pure music, combined with the neutral pictures and music (audio-visual) as a baseline. The material is a combination of the classic icons of these types of software, the combination of pictures and sounds, and the combination of the classic icons of the software and the associated cues. 
The polygraph production of Holland TMS132, consists of two parts: (1) DELL notebook computer, built-in AcqKnowledge3.9 software, is used to record the different channel waveform, skin conductance and skin electric sampling rate is set to sps32, blood volume pulse sampling rate is set to sps128, Suplab2.0 software, presentation the picture through the computer screen stimulation, with stereo headset when present at the same time with pictures and sound stimulation; (2) the BioTrace data acquisition system. This experiment uses this system to calculate the maximum, minimum, mean, variance, standard deviation, etc..

Experimental Design Single factor was used in the design, the picture (neutral pictures, six types of APP software icon) is the subject of the test, the presentation order of the image in the trial balance. The combination of pictures and sounds (neutral pictures and neutral music, six types of APP software images and the associated sound) for the test factors, the picture and the sound of the combination of the order in the trial balance.

Experimental Procedures The whole experiment was carried out in the laboratory of physiology and psychology. The experiment was conducted in five steps.

The first step: the subjects in the lab, fill in the personal information table, told they would do a physiological reaction experiment and the corresponding basic links, and tell them the experiment without any harm to them, only seriously on the line, at the beginning of the experiment, the first rest 3-5 minutes and keep the subjects the peace of mind, so their body temperature to maintain the normal temperature, the temperature of the laboratory is strictly controlled, maintained at 22 to 2 degrees Celsius. During the break, the wires will be connected, and the experiment will start after the break.

The second step: the subjects were asked to sit in front of the computer to maintain a comfortable posture, eye distance of $40 \mathrm{~cm}$, with physiological response sensor (BVP sensor, skin temperature, skin electric), with stereo headset, stay focused.

The third step: formal experimental stage. Formal experiment by A, B two links, A on behalf of the picture stimulus stage, $B$ represents the image and sound combination of the stimulus phase, the experiment uses ABBA way to balance, A, B stimulus presentation mode and time allocation as follows:

A: began to keep calm and relaxed (60s), instruction (30s), neutral pictures (10s), QQ (10s), Iqiyi icon icon (10s), happy diminshing music icon (10s), Taobao (10s), Alipay icon icon (10s), cool dog icon (10s), air screen the rest (20s).

B: the combination of neutral pictures and sounds to start (78S), QQ (10s), Iqiyi map acoustic sound (10s), happy diminshing music sound map (10s), Taobao (10s), sound (10s) to pay treasure map sound cool dog figure sound (10s).

The fourth step: the end of the experiment, removed from the sensor, giving small gifts.

Physiological Indexes Due to the limitation of experimental conditions, this experiment mainly three physiological indicators of skin, skin temperature, blood volume pulse recorded at the same time etc.. Three physiological indicators are as follows:

1 Skin temperature (Temp): in this experiment, the left middle finger of the subject is used to indicate the temperature. The skin temperature can reflect the changes of mood, the physiological response of the strong low temperature and high temperature reaction is weak.

2 Blood volume pulsation (BVP): the BVP sensor is tied to the left thumb to measure blood flow. BVP waveform can show the different characteristics of the heart beat cycle characteristics, when people are excited, surprised or frightened, the signal waveform will appear very crowded, which will lead to high BVP value. When the body is relaxed, the blood flow can be smooth to the end, waveform amplitude will increase significantly, then the value of BVP will be low.

3 Skin conductance response(SC): GSR electrodermal feedback instrument worn on the left hand index finger and ring finger. It is closely related to the secretion of sweat glands in the skin. When nervous or excited, the sympathetic nervous excitement, increased secretion of sweat, sweat on the skin increased, decreased skin resistance reaction of hand, caused by an increase in conductivity due to galvanic skin increased; conversely, when feeling calm and relaxed, sympathetic nerve decreased, reduce sweat secretion, skin conductivity decreases, cause skin electric value reduce. 
Physiological Response Data Acquisition Using SuperLab Pro software showed stimulation, using three indicators of BioTrace biofeedback instrument for measuring skin temperature, skin conductance and blood volume pulse, physiological response data collected off-line data was analyzed by AcqKnowledge software,

Calculate the mean value of A and B two stimulation mode under the skin temperature, skin conductance and blood volume pulse, and convert the data to SPSS18.0 for statistical analysis.

\section{Results and Analysis}

Analysis of the differences of emotional responses to app under different stimulation modes

Table 1 descriptive statistics of physiological indexes under different stimulation modes

\begin{tabular}{llcc} 
& & Mean & standard deviation \\
\hline Temp & Baseline & 32.659 & 1.879 \\
& Picture stimulus & 31.766 & 2.065 \\
& $\begin{array}{l}\text { Audiovisual } \\
\text { stimulation }\end{array}$ & 30.343 & 2.096 \\
BVP & Baseline & -27.767 & 11.346 \\
& Picture stimulus & -25.594 & 10.693 \\
& Audiovisual & -22.187 & 10.313 \\
& stimulation & & 1.173 \\
SC & Baseline & 2.117 & 1.160 \\
& Picture stimulus & 2.812 & 1.188 \\
& Audiovisual & 4.184 & \\
\hline
\end{tabular}

From table 1 the mean skin temperature can be seen in the two kinds of stimulation mode the temperature is lower than the baseline value, the combination of picture and sound under the stimulation of the skin temperature was higher than stumuli low blood volume pulse; in two kinds of stimulation mode values are higher than the baseline value, combined with one of the pictures and sound stimulation the blood volume pulse value than the picture stimulation mode is higher; in the two kinds of skin stimulation mode values were higher than the baseline value, combined with the picture and sound stimulation under the condition of the skin conductance value than picture stimulation mode to high. These are verified in the mean line graph.

The skin temperature stimulation and visual stimulation mode were lower than the baseline value, shows two kinds of stimulation mode of APP software are caused by physiological changes, skin temperature, skin temperature under visual stimulation than stumuli skin temperature, the physiological arousal under visual stimulation to stimulate better than single channel picture.

Two kinds of stimulation under the mode of APP software BVP values are higher than the baseline value, shows two kinds of stimulation patterns were the physiological changes caused by BVP, which stimulate the mode of the BVP value is greater than the picture stimulation mode value, indicating that the effect of audiovisual stimuli under the mode of APP software than the picture mode caused by physiological the reaction is better.

The picture stimulation and visual stimulation under the mode of APP software skin conductance values were higher than the baseline value, shows two kinds of stimulation patterns are caused by physiological changes in skin conductance, visual stimulation under the mode of skin conductance value than stumuli, that visual stimulation mode app software than the picture effect stimulation mode good. 
Table 2 Homogeneity test of variance of physiological indexes

\begin{tabular}{|c|c|c|c|c|}
\hline & & df1 & $\mathrm{df} 2$ & $\begin{array}{l}\text { Significance } \\
\text { level }\end{array}$ \\
\hline Temp & .448 & 2 & 150 & .640 \\
\hline BVP & . 190 & 2 & 150 & .827 \\
\hline $\mathrm{SC}$ & .047 & 2 & 150 & .954 \\
\hline
\end{tabular}

It can be seen from table 2 , the probability of $\mathrm{P}$ test of homogeneity of variance to calculate the value of the temperature value were 0.640 , blood volume pulse value 0.827 , skin 0.954 , its value was more than 0.05 in a given confidence level is 0.05 under the premise of the homogeneity of variance test, single factor analysis of variance can be further.

Table 3 Variance Analysis of emotional physiological responses under different stimulation modes

\begin{tabular}{|c|c|c|c|c|c|c|}
\hline & & $\begin{array}{l}\text { Sum of } \\
\text { squares }\end{array}$ & $\mathrm{df}$ & mean square & $\mathrm{F}$ & $\begin{array}{c}\text { Significanc } \\
\text { e level }\end{array}$ \\
\hline \multirow{4}{*}{$\begin{array}{l}\text { Tem } \\
\mathrm{p}\end{array}$} & Between & 139.220 & 2 & 69.610 & 17.126 & .000 \\
\hline & groups & & & & & \\
\hline & $\begin{array}{l}\text { Within the } \\
\text { group }\end{array}$ & 609.680 & 150 & 4.065 & & \\
\hline & Total & 748.900 & 152 & & & \\
\hline \multirow[t]{3}{*}{ BVP } & $\begin{array}{l}\text { Between } \\
\text { groups }\end{array}$ & 806.872 & 2 & 403.436 & 3.463 & .034 \\
\hline & $\begin{array}{l}\text { Within the } \\
\text { group }\end{array}$ & 17473.326 & 150 & 116.489 & & \\
\hline & Total & 18280.199 & 152 & & & \\
\hline \multirow[t]{3}{*}{$\mathrm{SC}$} & $\begin{array}{l}\text { Between } \\
\text { groups }\end{array}$ & 112.876 & 2 & 56.438 & 40.917 & .000 \\
\hline & $\begin{array}{l}\text { Within the } \\
\text { group }\end{array}$ & 206.900 & 150 & 1.379 & & \\
\hline & Total & 319.776 & 152 & & & \\
\hline
\end{tabular}

From table 3 the calculated temperature of $\mathrm{F}(2150)=17.126$, can be considered two kinds of stimulation mode under the app software due to physiological reactions and skin temperature significantly; blood volume pulse F $(2150)=3.463$, showed that two kinds of stimulation mode of APP software has obvious effects on the physiological indexes of the blood volume pulse skin; F $(2150)=40.917$, show the effects of two kinds of stimulation under the mode of APP software on galvanic skin significantly.

Using LSD method and Dunnett $\mathrm{C}$ for multiple comparisons, from table 2, the known variance is homogeneous, so the LSD method is applicable. By LSD multiple comparison indicated that the physiological indexes of temperature, compared with the baseline two kinds of stimulation patterns $\mathrm{P}$ values were less than 0.05 , compared with two baseline stimulation pattern stimulus pattern difference, shows two kinds of stimulation of the app software have caused a corresponding physiological response, including baseline and picture stimuli compared significantly. With the visual stimulus pattern significantly, and stimulate the picture stimulus pattern difference is also very significant; physiological index of blood volume pulse, baseline and picture stimuli no significant difference, compared with the mode of visual stimulation significantly, picture stimulation and visual stimulation compared no significant difference; physiological index of skin electric, and picture stimuli compared to the baseline obviously, compared with the visual stimulation pattern is significantly different, picture stimulation and visual stimulation is also very significant compared. 


\section{Discussion}

The background of this research is the app software which is closely related to the information era. In the use of APP software to obtain these entertainment and information, with visual, audio and visual stimuli. From the domestic and foreign research, it is known that most of the selected background materials are selected from the International Affective Picture System, which is different from the previous studies. The purpose of this experiment is to explore the impact of the intelligence age on the potential physiological responses of people, and to analyze the differences in the physiological responses of subjects with different genders and grades.

The experiment results prove the previous research, which stimulate the physiological response mode is better than single channel picture stimuli, the results of the experiment varied among the two indicators of skin temperature and skin conductance significantly, that stimulates the responses of subjects, and the two indexes and the body temperature of the human body, when excited when the temperature is reduced, increased sweating, resulting in two indicators at the same time significantly, no significant differences in the indexes of blood volume pulse, may be the two stages of the stimulus presentation time is short, not enough to cause greater fluctuations of heart beating, after the trial will try to extend the stimulus presentation time.

Research shows that more than half of college students daily use of mobile phone network for 2-4 hours, the mobile phone network occupies an important position in the students' study and life, the Internet often browse is mainly entertainment, including communication social software is the highest proportion, the characteristics of mobile phone network information dissemination, multi frequency image, interactive [14] it also make students when accepting network information has its own preference, the most common in the use of APP software is also open app interface icon, then contact the software in the process of using sound, this experiment also follow this step when using app, see the app icon will be stimulation of their physiological responses, if the software of voice prompts in the use of the app software, the software will further strengthen their students Physical reaction, some students may prefer to use the icon design and image appropriate to express the mood and tone software app software, when using the app software, with icons and sounds will also strengthen their physiological responses, individuals feel the excitement will also increase the frequency and time of use. So this experiment suggested that app software designers should not only design the classic image of the app icon to the app prompt carefully, after all, the two combine to make this software representative and easy to wake them using the desire to make the development more commercial value; for college students, or to control the use of mobile phone time, avoid the dependency on mobile phone.

\section{Conclusion}

Two kinds of stimulation under the mode of APP software have led to the individual physiological responses, including emotional reaction effect pictures and sound stimulus combination mode is better than the picture stimuli.

\section{Acknowledgement.}

This research was financially supported by the Key research project of humanities and social sciences of Anhui Provincial Department of Education (SK2016A0879). \&Horizontal scientific research project of Huangshan University.

\section{Reference}

[1] Yawei Zhao. Emotional design of mobile phone APP interface Shandong University, (2014) (in Chinese) 
[2] Ting Li. Research on APP interface design of smart phone based on user experience. Taiyuan University of Technology, (2015) (in Chinese)

[3] Huan Wang, the Yellow Sea, Wu Ming. The relationship between personality traits and college students' mobile phone dependence: the mediating effects of social anxiety Chinese. Journal of clinical psychology, , 22 (3): 447-450. (2014) (in Chinese)

[4] Yongzhi Jiang, white lily. College Students Internet dependence on mobile phone affect Alienation: [J]. psychological development and education, social support system, 2014, 30 (5): 540-549. (in Chinese)

[5] Zheng Zheng . The effect of picture stimuli on the priming effect of different emotional models [J]. chin J Sinica, 35 (6): 352-357.( 2003) (in Chinese)

[6] Hongyu Yang, Wenjuan Lin. Study on the positive and negative emotional responses induced by the International Affective Picture System in China [J]. Chinese Journal of behavioral medicine, 14 (): 1028-1030. (2005) (in Chinese)

[7] Jun Ren, Lu Huang, Zhenxin Zhang. Meditation makes people become peaceful. The emotional response of people to positive and negative emotional pictures can be reduced by meditation training [J]. Journal of psychology, 44:1339-1348. (2005) (in Chinese)

[8]. Yan Min Miao. stimulation effect of emotional autonomy physiological activities of [D]. research lab at Hunan Normal University, 2009(in Chinese)

[9] Baumgartner T, Esslen M, Jäncke L. From emotion perception to emotion experience: Emotions evoked by pictures and classical music[J]. International Journal of Psychophysiology, 60(1): 34-43.(2006)

[10] Mauri M, Cipresso P, Balgera A, et al. Why is Facebook so successful? Psychophysiological measures describe a core flow state while using Facebook[J]. Cyberpsychology, Behavior, and Social Networking, 2011, 14(12): 723-731. 